\title{
Engineering III-V nanowires for optoelectronics: from epitaxy to terahertz photonics
}

\author{
Hannah J. Joyce $^{* a}$, Chawit Uswachoke ${ }^{a}$, Sarwat A. Baig ${ }^{\text {a }}$, Stephanie O. Adeyemo ${ }^{\text {a }}$, Jessica L. \\ Boland $^{\mathrm{b}}$, Djamshid A. Damry ${ }^{\mathrm{b}}$, Christopher L. Davies ${ }^{\mathrm{b}}$, Jennifer Wong-Leung ${ }^{\mathrm{c}}$, H. Hoe Tan ${ }^{\mathrm{c}}$, \\ Jagadish, C. ${ }^{c}$, Laura M. Herz ${ }^{\mathrm{b}}$, and Michael B. Johnston ${ }^{\mathrm{b}}$ \\ aDepartment of Engineering, University of Cambridge, 9 JJ Thomson Avenue \\ Cambridge CB3 OFA, United Kingdom; \\ bDepartment of Physics, University of Oxford, Clarendon Laboratory, Parks Road, \\ Oxford OX1 3PU, United Kingdom; \\ 'Department of Electronic Materials Engineering, Research School of Physics and Engineering, \\ The Australian National University, Canberra ACT 2601, Australia
}

\begin{abstract}
Nanowires show unique promise as nanoscale building blocks for a multitude of optoelectronic devices, ranging from solar cells to terahertz photonic devices. We will discuss the epitaxial growth of these nanowires in novel geometries and crystallographic phases, and the use of terahertz conductivity spectroscopy to guide the development of nanowire-based devices. As an example, we will focus on the development of nanowire-based polarization modulators for terahertz communications systems.
\end{abstract}

Keywords: Nanowire, III-V, semiconductor, terahertz photonics, GaAs, epitaxy.

\section{INTRODUCTION}

Amongst semiconductor nanowires, III-V nanowires, such as GaAs and InP nanowires, are particularly promising for optoelectronic devices, ranging from solar cells ${ }^{1,2}$ to integrated photonic circuits ${ }^{3}$. Arguably one of the most promising fabrication techniques is metalorganic chemical vapour deposition (MOCVD), using Au nanoparticles to direct anisotropic nanowire growth. This fabrication process enables the growth of axial and radial (core-shell) heterostructures (Figure 1a and $b$ ), and the growth of unconventional crystal phases, with the tight control needed for the manufacture of nanowirebased devices.

In addition to the ability to fabricate nanowires with high precision, a detailed understanding of the electronic properties of nanowires is imperative for the development of novel nanowire-based devices. ${ }^{4}$ As a contact-free method of assessing ultrafast carrier dynamics and transport, terahertz conductivity spectroscopy is ideally suited for electrical characterisation of nanowires. Key electrical parameters, such as charge carrier mobility, ionised dopant density, charge carrier lifetime and surface recombination velocity, are readily measurable with terahertz conductivity spectroscopy. ${ }^{5}$

Finally, nanowires show unique promise for optoelectronic devices operating in the terahertz spectral region, such as photoconductive detectors, terahertz emitters and modulators. ${ }^{6,7}$ For example, films of aligned GaAs nanowires act as THz polarizers that can be modulated on picosecond timescales via photoexcitation. These polarization modulators have immense potential for $\mathrm{THz}$ communications. ${ }^{7}$

*hannah.joyce@eng.cam.ac.uk; phone +44 1223748379 
This paper discusses the how the growth of novel and complex III-V nanowires is achieved through MOCVD, how terahertz conductivity spectroscopy reveals the fascinating properties of these nanowires, and how these measurements are guiding the development of optoelectronic devices.

\section{EXPERIMENTAL METHODS}

\subsection{Epitaxy of nanowires}

The ability to fabricate nanowires with tightly defined electrical, optical and structural properties is a prerequisite for the development of nanowire-based optoelectronic devices. MOCVD, using Au nanoparticles to drive nanowire growth, is one of the most promising and well-studied techniques for nanowire growth. In this process, the Au nanoparticles collect reaction species and drive highly anisotropic nanowire epitaxy via a vapour-liquid-solid or vapour-solid-solid growth technique. ${ }^{8}$ This approach offers high reproducibility, tight control over nanowire properties and scalability for industrial mass fabrication. A number of growth parameters can be tuned to optimise nanowire growth, ${ }^{9,10}$ including temperature and the flow rates of group III and group V precursor species. For example, by choosing appropriate growth temperatures and V/III flow ratios, nanowire crystal structure can be tuned from twin free zinc-blende and stacking fault-free wurtzite, ${ }^{11}$ and their charge carrier lifetimes and charge carrier mobilities can be enhanced ${ }^{12}$.

Here, growths were performed in an AIXTRON 200/4 horizontal flow MOCVD reactor at a pressure of $100 \mathrm{mbar}$ with $\mathrm{H}_{2}$ carrier gas at a total gas flow rate of $15 \mathrm{slm}$. The group III precursors were TMGa, TMIn and TMAl, and the group V precursors were $\mathrm{AsH}_{3}$ and $\mathrm{PH}_{3}$. Nanowires typically grow in the [111]B crystallographic direction and are free standing on the growth substrate. The nanowires were grown on epi-ready (111)B-oriented substrates to achieve nanowires that are vertical on the growth substrate, or on (100)-oriented substrates to achieve angled nanowires growing along two equivalent [111]B directions at $35.3^{\circ}$ to the plane of the substrate (Figure 1c). ${ }^{7}$ Monodisperse colloidal Au nanoparticles of a particular size $(20,30,50$ or $80 \mathrm{~nm}$ diameter) were used to control the diameter of the grown nanowires. GaAs nanowires and GaAs nanowire cores were grown using a two-temperature procedure as published previously. ${ }^{13}$ For GaAs/AlGaAs core-shell nanowires, AlGaAs shells were grown at $750{ }^{\circ} \mathrm{C}$ to passivate the surface of the GaAs. ${ }^{14}$

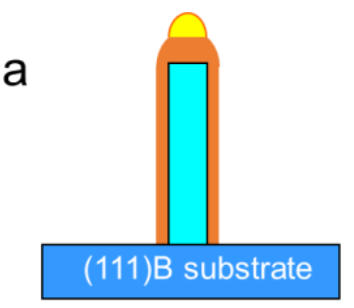

Radial heterostructure

(core/shell nanowire)

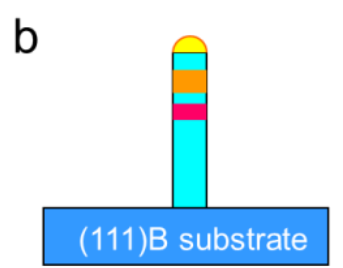

Axial heterostructure

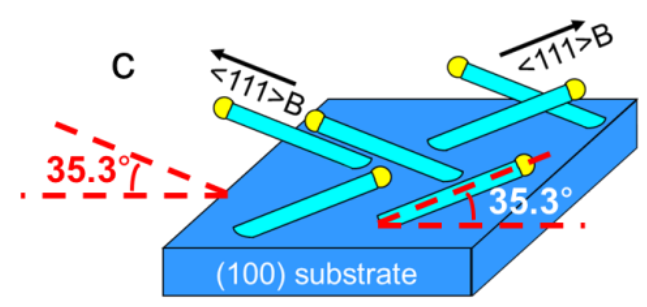

Angled nanowires

Figure 1. Schematic diagrams of $(a, b)$ vertical nanowires grown on (111)B substrates and (c) angled nanowires grown on a (100) substrate. Also illustrated are (a) a core-shell heterostructure nanowire (radial heterostructure) and (b) an axially heterostructured nanowire. 


\subsection{Measurement of nanowire photoconductivity by optical pump-terahertz probe spectroscopy}

After growth, vertical nanowires were transferred to z-cut quartz by rubbing the as-grown substrates against the quartz substrates. Angled nanowires were embedded in a $5 \mu \mathrm{m}$ thick layer of Parylene $\mathrm{C}$, which was then peeled from the growth substrate to yield nanowires embedded in a free-standing Parylene $\mathrm{C}$ membrane. Both Parylene $\mathrm{C}$ quartz are suitable as the host matrix/substrate as they are highly transparent to $\mathrm{THz}$ radiation and do not exhibit any photoconductivity response.

Terahertz generation, photoexcitation of the sample, and terahertz detection were achieved using an amplified Ti:Sapphire laser generating $35 \mathrm{fs}$ pulses centred at $800 \mathrm{~nm}$ at a $5 \mathrm{kHz}$ repetition rate and $4 \mathrm{~W}$ average power. The laser beam was split into three paths: (i) $200 \mathrm{~mJ} /$ pulse was used to generate the $\mathrm{THz}$ probe pulse via optical rectification in a $2 \mathrm{~mm} \mathrm{GaP}$ crystal, (ii) $1.6 \mathrm{~mJ} /$ pulse was used as a gate for electro-optic detection of the transmitted $\mathrm{THz}$ pulse using a $200 \mu \mathrm{m} \mathrm{GaP}$ crystal, and (iii) as the optical pump to photoexcite the sample at fluences between 1 and $280 \mathrm{~mJ} / \mathrm{cm}^{2}$. At the sample position, the optical pump beamwidth was approximately 10 times larger than the $\mathrm{THz}$ probe beamwidth, so that the terahertz probe measured an area of approximately constant photoexcited carrier density. The THz electric field, $E$, was detected using a balanced photodiode circuit connected to a lock-in amplifier referenced to a chopper $(2.5 \mathrm{kHz})$ in the $\mathrm{THz}$ generation beam. A second lock-in amplifier was used to detect the photoinduced change in terahertz electric field, $\Delta E$, referenced to a second chopper $(125 \mathrm{~Hz})$ in the optical pump beam. To investigate the $\mathrm{THz}$ spectral response of the material as a function of time after photoexcitation, we varied the delay between the optical pump, terahertz probe and gate pulses. All measurements were performed at room temperature.

\section{RESULTS AND DISCUSSION}

\subsection{Terahertz photoconductivity in semiconductor nanowires}

Semiconductor nanowires typically exhibit a pronounced Lorentzian response in their terahertz photoconductivity spectra, ${ }^{15}$ which is defined by the function

$$
\Delta \sigma(\omega)=\frac{n q^{2}}{m^{*}} \frac{i \omega}{\omega^{2}-\omega_{0}^{2}+i \omega \gamma},
$$

where $\Delta \sigma$ is the photoconductivity as a function of frequency $\omega, n$ is the photoexcited electron density, $q$ is the electronic charge, $m^{*}$ is the electron effective mass in GaAs and $\omega_{0}$ is the resonant frequency. A typical spectrum obtained from an ensemble of GaAs nanowires is illustrated in Figure 2. This response arises from longitudinal plasmon oscillations, or equivalently by considering Maxwell-Garnett effective medium theory together with the Drude model. Using the latter approach, ${ }^{16}$ it can be shown that the resonant frequency is given by

$$
\omega_{0}=\sqrt{\frac{(1-f)}{B} \frac{n q^{2}}{m^{*} \epsilon_{0}}},
$$

where $B$ is a constant related the geometry of the nanowires, the fractional volume occupied by the nanowires (fill factor), and the dielectric constants of both the nanowires and the host medium. Equation (2) illustrates the dependence of $\omega_{0}$ on the carrier density $n$, which is characteristic of plasmon resonance. ${ }^{15,17}$

Photoconductivity spectra were fitted with Lorentzian functions (Equation 1). The electron scattering rate, $\gamma$, used as a fitting parameter, was readily converted to electron mobility via

$$
\mu=\frac{q}{m_{e}^{*} \gamma}
$$

where $m_{e}{ }^{*}$ is the electron effective mass and $q$ is the electronic charge. Thus, the fits allowed extraction of electron mobility for each sample. 


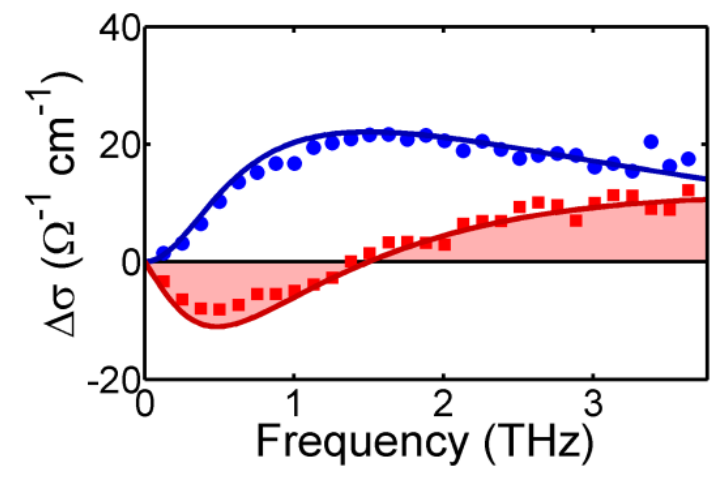

Figure 2. Photoconductivity spectra of GaAs nanowires with average diameters of $50 \mathrm{~nm}$, taken at $1 \mathrm{ps}$ after photoexcitation with a photoexcitation fluence of $10 \mu \mathrm{J} \mathrm{cm}^{-2}$. The symbols are the measured data and the lines are the fitted plasmon responses. The real (blue circles and lines) and imaginary (red squares and lines) components of the conductivity are plotted.

Unpassivated GaAs nanowires featured diameter-dependent electron mobilities (400 to $2100 \mathrm{~cm}^{2} \mathrm{~V}^{-1} \mathrm{~s}^{-1}$ ) and diameterdependent charge carrier lifetimes ( 1 to $5 \mathrm{ps}$ ) at room temperature. ${ }^{15}$ The electron mobility decreased significantly with decreasing nanowire diameter as charge carriers experience increased scattering at nanowire surfaces. Reducing the diameter from $50 \mathrm{~nm}$ to $30 \mathrm{~nm}$ reduced the electron mobility by almost $50 \%$. ${ }^{16}$ The photoconductivity dynamics were dominated by trapping at surface states, and the trapping rate was highest for the nanowires of narrowest diameter. These surface states were saturable, as evidenced by the non-exponential photoconductivity decays and the slowing of trapping with increasing fluence and increasing time after photoexcitation. ${ }^{12}$ The maximum surface recombination velocity, which occurs in the limit of all traps being empty, was calculated to be approximately $10^{6} \mathrm{~cm} / \mathrm{s} .{ }^{16}$

Both charge carrier lifetime and mobility are strongly influenced by the surface of the GaAs nanowires, pointing to the importance of surface passivation. We investigated and optimised the growth of AlGaAs shells to achieve surface passivation and thereby enhance charge carrier lifetimes and mobilities. By increasing AlGaAs shell thickness, the carrier lifetimes and mobilities both improved significantly. Optimized GaAs/AlGaAs core-shell nanowires exhibited electron mobilities up to $3000 \mathrm{~cm}^{2} \mathrm{~V}^{-1} \mathrm{~s}^{-1}$, which is over $65 \%$ of the electron mobility typical of high quality undoped bulk GaAs at comparable carrier densities. A drastic improvement in photoconductivity lifetime, reaching 1.6 ns was observed in the optimised core-shell nanowires. This lifetime is almost three orders of magnitude greater than that observed in bare unpassivated GaAs nanowires. Optimised core-shell nanowires have since been used to demonstrate nanowire lasers operating at room temperature ${ }^{18}$ and ultrafast photoconductive terahertz detection. ${ }^{6}$

\subsection{Terahertz photonic devices based on aligned GaAs nanowires}

Unpassivated GaAs nanowires are strong candidates for ultrafast optically switchable $\mathrm{THz}$ polarizers as they exhibit (i) large broadband photoconductivity in response to photoexcitation due to their high optical absorption coefficients and high electron mobilities $\left(\sim 1000 \mathrm{~cm}^{2} \mathrm{~V}^{-1} \mathrm{~s}^{-1}\right),{ }^{16}$ (ii) rapid $(<5 \mathrm{ps})$ photoconductivity decays due to their short charge carrier lifetimes and high surface recombination velocity ${ }^{15}$ and (iii) polarization-dependent absorption of both visible/NIR radiation $^{19}$ and $\mathrm{THz}$ radiation ${ }^{5}$ due to the anisotropic nanowire geometry. In principle, GaAs nanowires arranged in a wiregrid configuration and rendered conductive by photoexcitation, will modulate the transmission of THz radiation.

$\mathrm{THz}$ polarisers were constructed by embedding the aligned angled nanowires (grown on (100) substrates) in a flexible membrane of Parylene $\mathrm{C}$ polymer. Films of nanowires-in-polymer were then laminated together to create multilayer films up to 14 layers thick. The design mimics conventional wire-grid polarizers, but uses the semiconducting nature of the nanowires with conduction that can be switched "on" via photoexcitation (Figure 3). Their operation is similar to that of nanostructured polarisers based on semiconducting carbon nanotubes. ${ }^{20}$ When photoexcited by an ultrashort optical pulse, nanowires selectively absorb the component of the incident $\mathrm{THz}$ pulse $(0.1$ to $4 \mathrm{THz})$ polarized parallel to the nanowire axes. 

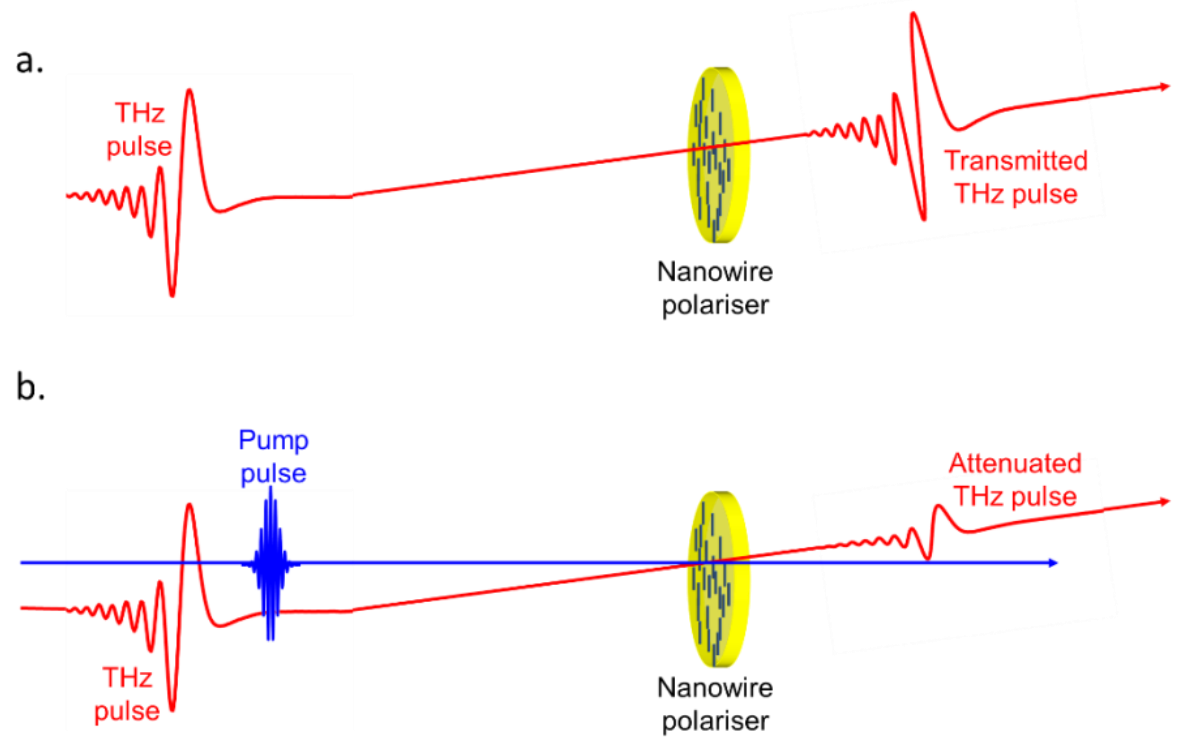

Figure 3. Schematic diagram illustrating the operation of the THz polarization modulator. (a) Without photoexcitation, the $\mathrm{THz}$ pulse is transmitted through the polarizer with minimal absorption (transmission). (b) Photoexcitation renders the nanowires conductive to absorb the component of the THz pulse oriented parallel to the nanowire axis (attenuation).

To characterise the polarisers, we employed optical pump-THz probe spectroscopy and varied the polarization of the optical pump beam to selectively photoexcite nanowires oriented parallel to the pump polarisation. The $\mathrm{THz}$ transmission followed a cosine-squared relationship with pump polarisation angle, as expected from Malus's law. ${ }^{7}$ We increased the areal density of nanowires by laminating layers, and thereby increased the modulation depth and extinction coefficient of the polariser. The best performance was observed for the 14-layer polarizer, which exhibited a modulation depth of $-8 \mathrm{~dB}$. This is significantly better than ultrafast polarizers based on carbon nanotubes. ${ }^{20}$ After photoexcitation, the transient $\mathrm{THz}$ absorption decayed on a picosecond time scale, and this property allows ultrafast modulation of the $\mathrm{THz}$ transmission. ${ }^{7}$ The nanowire-grid polarizer combines a large modulation depth, broad bandwidth and picosecond switching. These properties make it ideal as a modulator in future high-speed, picosecond $\mathrm{THz}$ communication.

\section{CONCLUSIONS}

Nanowire devices have enormous advantages over their conventional planar counterparts. The key to achieving state-ofthe-art nanowire devices is a rapid and accurate feedback pathway between nanowire growth and nanowire electrical characterisation. We have described how terahertz conductivity spectroscopy is yielding high throughput, highly accurate measurements of key electrical parameters, such as charge carrier mobilities, lifetimes and surface recombinations velocities. These measurements are guiding the development of novel nanowire-based devices, such as GaAs nanowirebased THz polarisation modulators. These modulators feature large modulation depths, broad bandwidth in the THz range, and picosecond switching speeds, suggesting their strong potential in future terahertz communications systems. 


\section{ACKNOWLEDGEMENTS}

The authors acknowledge financial support from the European Research Council (ERC Starting Grant ACrossWire), the Engineering and Physical Sciences Research Council (UK), the Australian Research Council, and the Australian National Fabrication Facility.

\section{REFERENCES}

[1] Wallentin, J., Anttu, N., Asoli, D., Huffman, M., Aberg, I., Magnusson, M. H., Siefer, G., Fuss-Kailuweit, P., Dimroth, F., Witzigmann, B., Xu, H. Q., Samuelson, L., Deppert, K. and Borgstrom, M. T., "InP nanowire array solar cells achieving $13.8 \%$ efficiency by exceeding the ray optics limit," Science (80-. ). 339(6123), 1057-1060 (2013).

[2] Krogstrup, P., Jørgensen, H. I., Heiss, M., Demichel, O., Holm, J. V, Aagesen, M., Nygard, J. and Fontcuberta i Morral, A., "Single-nanowire solar cells beyond the Shockley-Queisser limit," Nat. Photon. 7(4), 306-310 (2013).

[3] Pauzauskie, P. J. and Yang, P., "Nanowire photonics,” Mater. Today 9(10), 36-45 (2006).

[4] Alexander-Webber, J. A., Groschner, C. K., Sagade, A. A., Tainter, G., Gonzalez-Zalba, M. F., Pietro, R. Di, Wong-leung, J., Tan, H. H., Jagadish, C., Hofmann, S. and Joyce, H. J., "Engineering the Photoresponse of InAs Nanowires," ACS Appl. Mater. Interfaces 9, 43993-44000 (2017).

[5] Joyce, H. J., Boland, J. L., Davies, C. L., Baig, S. and Johnston, M. B., “A review of the electrical properties of semiconductor nanowires: Insights gained from terahertz conductivity spectroscopy.," Semicond. Sci. Technol. 31(10), 103003 (2016).

[6] Peng, K., Parkinson, P., Fu, L., Gao, Q., Jiang, N., Guo, Y.-N., Wang, F., Joyce, H. J., Boland, J. L., Tan, H. H., Jagadish, C. and Johnston, M. B., "Single nanowire photoconductive terahertz detectors," Nano Lett. 15(1), 206$210(2015)$

[7] Baig, S. A., Boland, J. L., Damry, D. A., Tan, H. H., Jagadish, C., Joyce, H. J. and Johnston, M. B., “An Ultrafast Switchable Terahertz Polarization Modulator Based on," Nano Lett. 17, 2603-2610 (2017).

[8] Jiang, N., Wong-Leung, J., Joyce, H. J., Gao, Q., Tan, H. H. and Jagadish, C., "Understanding the true shape of Au-catalyzed GaAs nanowires," Nano Lett. 14(10), 5865-5872 (2014).

[9] Joyce, H. J., Gao, Q., Hoe Tan, H., Jagadish, C., Kim, Y., Zou, J., Smith, L. M., Jackson, H. E., Yarrison-Rice, J. M., Parkinson, P. and Johnston, M. B., "III-V semiconductor nanowires for optoelectronic device applications," Prog. Quant. Electron. 35, 23-75 (2011).

[10] Joyce, H. J., Gao, Q., Wong-Leung, J., Kim, Y., Tan, H. H. and Jagadish, C., “Tailoring GaAs, InAs, and InGaAs nanowires for optoelectronic device applications,” IEEE J. Sel. Top. Quantum Electron. 17(4), 766-778 (2011).

[11] Joyce, H. J., Wong-Leung, J., Gao, Q., Hoe Tan, H. and Jagadish, C., "Phase perfection in zinc blende and wurtzite III- V nanowires using basic growth parameters,” Nano Lett. 10(3), 908-915 (2010).

[12] Parkinson, P., Joyce, H. J., Gao, Q., Tan, H. H., Zhang, X., Zou, J., Jagadish, C., Herz, L. M. and Johnston, M. B., "Carrier lifetime and mobility enhancement in nearly defect-free core-shell nanowires measured using timeresolved terahertz spectroscopy," Nano Lett. 9(9), 3349-3353 (2009).

[13] Joyce, H. J., Gao, Q., Tan, H. H., Jagadish, C., Kim, Y., Zhang, X., Guo, Y. and Zou, J., “Twin-free uniform epitaxial GaAs nanowires grown by a two-temperature process," Nano Lett. 7(4), 921-926 (2007).

[14] Jiang, N., Parkinson, P., Gao, Q., Breuer, S., Tan, H. H., Wong-Leung, J. and Jagadish, C., "Long minority carrier lifetime in Au-catalyzed GaAs/AlxGa1-xAs core-shell nanowires,” Appl. Phys. Lett. 101(2), 23111 (2012).

[15] Joyce, H. J., Docherty, C. J., Gao, Q., Tan, H. H., Jagadish, C., Lloyd-Hughes, J., Herz, L. M. and Johnston, M. 
B., "Electronic properties of GaAs, InAs and InP nanowires studied by terahertz spectroscopy," Nanotechnology 24, 214006 (2013).

[16] Joyce, H. J., Boland, J. L., Davies, C. L., Joyce, H. J., Baig, S. A. and Parkinson, P., “The influence of surfaces on the transient terahertz conductivity and electron mobility of GaAs nanowires," J. Phys. D Appl. Phys. 50, 224001 (2017).

[17] Joyce, H. J., Wong-Leung, J., Yong, C.-K., Docherty, C. J., Paiman, S., Gao, Q., Tan, H. H., Jagadish, C., LloydHughes, J., Herz, L. M. and Johnston, M. B., "Ultralow surface recombination velocity in InP nanowires probed by terahertz spectroscopy,” Nano Lett. 12(10), 5325-5330 (2012).

[18] Saxena, D., Mokkapati, S., Parkinson, P., Jiang, N., Gao, Q., Tan, H. H. and Jagadish, C., “Optically pumped room-temperature GaAs nanowire lasers," Nat. Photon. 7(12), 963-968 (2013).

[19] Titova, L. V., Hoang, T. B., Jackson, H. E., Smith, L. M., Yarrison-Rice, J. M., Kim, Y., Joyce, H. J., Tan, H. H. and Jagadish, C., "Temperature dependence of photoluminescence from single core-shell GaAs-AlGaAs nanowires," Appl. Phys. Lett. 89(17), 173126 (2006).

[20] Docherty, C. J., Stranks, S. D., Habisreutinger, S. N., Joyce, H. J., Herz, L. M., Nicholas, R. J. and Johnston, M. B., “An ultrafast carbon nanotube terahertz polarisation modulator,” J. Appl. Phys. 115, 203108 (2014). 